\title{
1 Sexualised drug use in people attending sexual health clinics in England
}

2 Hamish Mohammed $^{1 *}$, John Were ${ }^{1}$, Carina King ${ }^{1,2}$, Martina Furegato ${ }^{1}$, Anthony Nardone ${ }^{1}$

3 and Gwenda Hughes ${ }^{1}$ on behalf of the GUMCADv3 Steering Group**

$4 \quad 1$ - HIV \& STI Department, Public Health England, London, UK

52 - Research Department of Infection and Population Health, University College London,

6 London, UK

7

$8 \quad$ * Corresponding author

9 Hamish Mohammed, PhD, MPH

10 Principal STI Prevention \& Surveillance Scientist, HIV \& STI Department

11 National Infection Service

12 Public Health England

1361 Colindale Avenue

14 London, UK

15 NW9 5EQ

16 hamish.mohammed@phe.gov.uk

17 Tel: 02083276403

** The members of the GUMCADv3 Steering Group are as follows: Afra Barrett, Jackie Cassell, Laura Clark, Claudia Estcourt, lain Galloway, Nigel Field, Patti Green, James Hardie, Leigh Holmes, Peter Horne, Gwenda Hughes, Hamish Mohammed, Monty Moncrieff, Anthony Nardone, David Phillips, David Stuart, Ann Sullivan and Sonali Wayal.

Keywords: drug misuse; injecting drug use; surveillance; gay men

Word count: 299 (limit: 300) 
Recent evidence highlights an increase in 'chemsex', the use of recreational drugs during sex, in men who have sex with men (MSM) and an association with risky sexual behaviours and outbreaks of sexually transmitted infections (STIs). ${ }^{1}$ However, the extent of sexualised drug use in people attending sexual health clinics (SHCs) is unknown.

STI surveillance in England is performed by Public Health England (PHE) using a disaggregated patient-level dataset of all diagnoses and services at SHCs. ${ }^{2}$ This is a minimum dataset with key demographic and clinical variables, but lacks behavioural data. To address this gap, PHE piloted a surveillance enhancement to collect behavioural data, including sexualised drug use (an affirmative response to 'were you under the influence of recreational drugs [before or during sex] with any partner in the last 3 months?'). A pilot in six SHCs throughout England took place from August 2013 to April 2014; at each clinic, attendees' behavioural data were collected for all new patient episodes.

Complete data on 8,741 attendances were submitted (48.5\% of eligible attendances). Overall, the proportion where sexualised drug use was reported was $6.6 \%$, ranging from $4.1 \%$ in heterosexual women to $12.1 \%$ in MSM. Among the 519 MSM who reported at least one sex partner in the last three months, the most commonly reported drugs used before/ during sex were mephedrone (10.4\%), $\gamma$-hydroxybutyrate/ $\gamma$-butyrolactone (GHB/GBL, 7.1\%) and cannabis (6.7\%). Chemsex is usually associated with mephedrone, crystal

These preliminary data suggest that sexualised drug use is commonly reported by SHC attendees, especially MSM, and highlight the utility of monitoring drug use at SHCs to identify local needs and plan care pathways for appropriate treatment services.

Acknowledgements: The authors would like to thank the staff at the following clinics for their contributions to this pilot (contact persons are listed for each clinic): 56 Dean St. (Drs. Alan McOwan and Sheel Patel); Clare Simpson Clinic (Ms. Jacinta Ryan); Croydon Sexual Heath Centre (Dr. David Phillips); Claude Nicol Centre (Dr. Gillian Dean and Ms. Catherine Hendrickx); THT/Brook Bedford (Mr. Phillip Mason and Ms. Tracey Tachikawa); and YorSexualHealth (Dr. Ian Fairley and Ms. Corinna Dass).

We declare no competing interests. 
59 Contributions: $\mathrm{GH}$ and $\mathrm{AN}$ conceived the pilot, and HM contributed to its design and

60 coordination. The GUMCADv3 Steering Group was responsible for designing and finalising

61 the data specification for the pilot. HM wrote the first draft of the manuscript and performed

62 the analysis with JW and CK. All authors read, critically reviewed and approved the final

63 version of the manuscript for publication. 
1. Bourne A, Reid D, Hickson F, et al. "Chemsex" and harm reduction need among gay men in South London. International Journal of Drug Policy 2015;26(12):1171-76. transmitted infections using mandatory electronic clinical reporting: the genitourinary medicine clinic activity dataset, England, 2009 to 2013. Euro Surveill. 2014;19(48):pii=20981. Available online: http://www.eurosurveillance.org/ViewArticle.aspx?Articleld=20981. Accessed 17th May 2016. 